\title{
FIB Damage Reduction Technique in TEM Membrane Using Triple Beam
}

\section{System}

\author{
S. Sadayama, H. Takahashi, K. Iwasaki and T. Fujii
}

SII NanoTechnology Inc., 36-1, Takenoshita, Oyama-cho, Sunto-gun, Shizuoka, 410-1393, JAPAN

\section{Introduction}

Focused Ion Beam (FIB) system has been used widely to make samples for Transmission Electron Microscopy(TEM) system. And recent application requires making a thinner membrane than previous one for a FIB system. Therefore, atomic level damages which are generated into the membrane during a FIB process become a real and a substantive issue, especially a $30 \mathrm{kV}$ energy FIB process makes them so much.

We have reported our double beam system which can make good TEM membranes with strict monitoring of the SEM in LSITS 2004, JAPAN.[1] In this study, we introduce a triple beam system, SMI3050TB, which is an argon (Ar) ion column is attached to our double beam system. The Ar ion column can reduce the FIB damages into TEM membrane dramatically. One of the advantages of the SMI3050TB is geometry of the columns. The all column aim the point in the main chamber. So operators can treat all processes for TEM preparation at the point easily.

Advantages of the Triple Beam System

Fig. 1 shows the geometry of the SMI3050TB.

The FIB column, the SEM column and the Ar column are mounted to aim the same point in the chamber as the Fig. 1 shown. This geometry design makes all the processes completed at the point with monitoring of SEM. The Ar column is mounted parallel to membrane sidewalls. The purpose of the Ar column geometry is that not only an incidence angle of the Ar beam can be slight, but also the processed membrane side can face the SEM. It informs the progress of the Ar beam process to operators.

Incidence angle of the Ar beam

An incidence angle of the Ar beam can be set precisely on our GUI software. The angle is determined by not only the stage rotation but also the stage tilt due to the column geometry. It bothers operators to determine the angle. So our software allows the operators only to input the number of an incidence angle. Then software calculates and drives both the rotation and the tilt stage immediately.

Examination of FIB Damage Reduction in Silicon

We investigated the damage reduction effect using the Ar beam as following method. A broken silicon wafer was used as a sample. A region of 10 micron width was milled by $30 \mathrm{kV}$ Ga ions and irradiated with $1 \mathrm{kV}$ Ar beam for 8 minutes on an edge of the broken silicon wafer. After a passivation film was capped on the region, a lamella of the region was made perpendicular to the silicon surface. Fig. 2 shows the result; Silicon crystals which have dark contrast on the bottom side and the passivation film which has bright contrast on the top side can be observed. 
The layer which has the intermediate contrast is the damage layer caused by the Ar beam irradiation. The thickness of the damage layer measures approximately $2 \mathrm{~nm}$. We have examined a thickness of that using $30 \mathrm{kV}$ Ga as same manner. It was $25 \mathrm{~nm}$.; This result proves to achieve a new level of the high quality TEM sample.

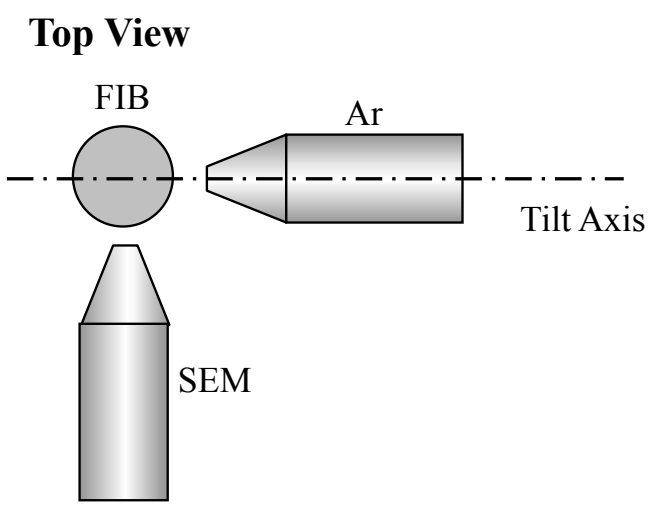

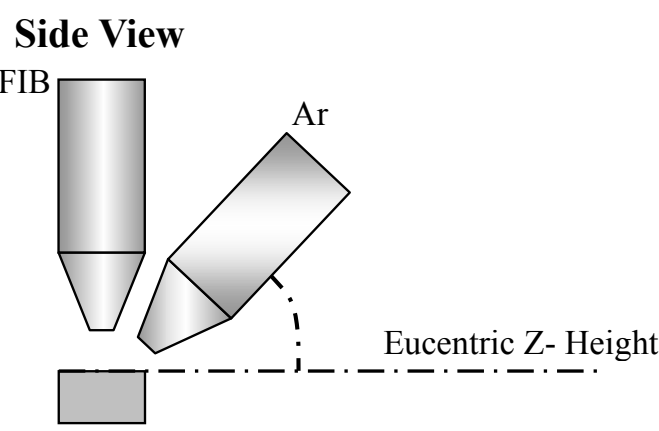

$47.5 \mathrm{deg}$. $/ 50 \mathrm{mmChamber}$ 55 deg. $/ 200300 \mathrm{mmChamber}$

Fig. 1. The geometry of the SMI3000TB series.

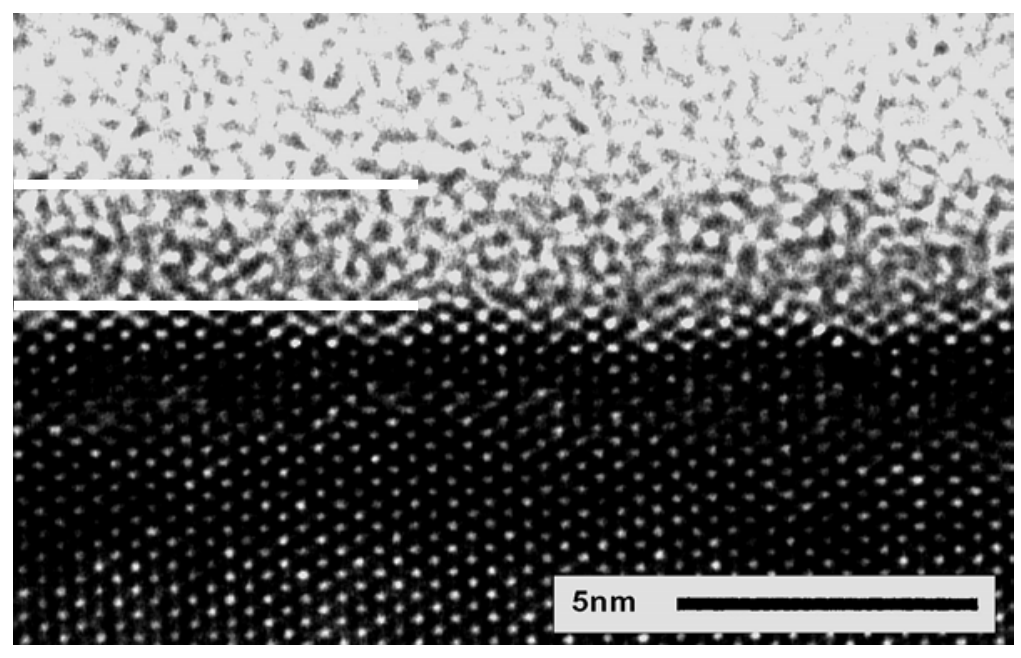

Fig. 2. HRTEM image of a silicon membrane after the $1 \mathrm{kV}$ Ar ion beam treatment. The thickness of damage layer of $2 \mathrm{~nm}$ can be seen. (It is shown between white lines in the figure.)

\section{References}

[1] I. Nakatani et al., LSI Testing Symposium2004 proceeding (2004) 